How to reference this article Kowalczyk-Cantoro, D. (2021). Le prime traduzioni polacche delle poesie italiane di Pietro Bembo. Italica Wratislaviensia, 12(2), 111-128.

DOI: http://dx.doi.org/10.15804/IW.2021.12.2.06

\author{
Daria Kowalczyk-Cantoro \\ Uniwersytet Wrocławski, Polonia \\ daria.kowalczyk@uwr.edu.pl \\ ORCID: 0000-0001-6768-8063
}

\title{
LE PRIME TRADUZIONI POLACCHE DELLE POESIE ITALIANE DI PIETRO BEMBO
}

\author{
THE FIRST POLISH TRANSLATIONS \\ OF PIETRO BEMBO'S ITALIAN POETRY
}

\begin{abstract}
The present article aims to analyse the $20^{\text {th }}$-century Polish translations of Pietro Bembo's work. Although Bembo was one of the main representatives of the Italian Renaissance and a prolific writer, of all his lyric poetry, only six songs from the collection Rime, a short poem from the dialogue Gli Asolani, and recently the Stanze have been fully translated. We owe most of the earlier translations to Julia Dickstein-Wieleżyńska, an important populariser of Italian culture in the interwar period. Two poems were translated by Maria Grossekowa, a poet, publicist, and feminist of the early $20^{\text {th }}$ century. The small fragments of Bembo's sonnets translated by Edward Porębowicz are also worthy of mention. The article focuses on the metric-formal and semantic-lexical analysis of DicksteinWieleżyńska's versions and examines the techniques used by the translator. Dickstein-Wieleżyńska's translations are quite equivalent semantically, and although she introduces some reduction or amplification, she does so without upsetting the semantic dominant. Moreover, it has been noted that, in her translations, Dickstein-Wieleżyńska often uses terms that refer to an idea of brightness, which also characterises her own poetic writing. Since Bembo is considered the pioneer of Petrarchism, the analysis of the Polish translations of Bembo's poems is deepened through comparisons with some versions of Petrarch's poems translated by Felicjan Faleński and published in 1881.
\end{abstract}

Keywords: translation, Bembo, Renaissance, Dickstein-Wieleżyńska, Grossekowa 


\section{INTRODUZIONE}

$\mathrm{M}$ algrado Pietro Bembo sia stato uno dei principali rappresentanti del Rinascimento italiano e possa essere considerato uno scrittore prolifico, i traduttori polacchi si sono cimentati con le sue opere in poche occasioni. Con ogni probabilità questo disinteresse per Bembo poggiava su un pregiudizio storico-letterario, ovvero quello di considerarlo un mero rappresentante del petrarchismo. Dunque un autore incapace di una sua originalità. Integralmente sono stati tradotti solo sei $^{1}$ componimenti poetici della raccolta Rime e, ultimamente, le Stanze (Bembo, 2019). Le prime versioni polacche della poesia bembiana in italiano sono state pubblicate soltanto alla fine del XIX secolo, quando di rado si traducevano poeti italiani delle epoche antiche, ad eccezione di Dante e Petrarca, il cui Canzoniere tradotto da Felicjan Faleński venne pubblicato nel 1881. Tra le prime antologie e storie della letteratura apparse all'epoca spiccano i quattro volumi di Dzieje literatury powszechnej (Storia della letteratura mondiale) (1883-1898) di cui fa parte il volume di Edward Porębowicz sulla letteratura italiana. Lo studioso ha illustrato la cultura letteraria della penisola italica traducendo per l'occasione diversi componimenti di vari autori. Sebbene non abbia tradotto integralmente nessun componimento del poeta veneziano, ha inserito nella sua monografia le proprie versioni di brevi frammenti di due sonetti (XII e XX) delle Rime.

Nel primo Novecento, insieme allo sviluppo degli studi in ambito romanzo, in Polonia cresce notevolmente il numero di traduttori della poesia italiana. Sono attive celebri traduttrici quali Maria Konopnicka, Waleria Marrené Morzkowska o Wilhelmina Zyndram-Kościałkowska. La poetessa, pubblicista e femminista Marja Grossekowa (GrossekKorycka) ha reso in polacco due sonetti di Bembo (il LX e il CLI) pub-

${ }^{1}$ La più completa bibliografia delle traduzioni polacche della poesia italiana elenca cinque componimenti della raccolta Rime (Miszalska, Gurgul, Surma-Gawłowska \& Woźniak, 2007, p. 144), senza prendere in considerazione la canzone La mia leggiadra e candida angioletta della stessa raccolta tradotta da Julia Dickstein-Wieleżyńska e pubblicata nel 1931 su Przeglad Humanistyczny. 
blicandoli su Panteon literatury wszechświatowej nel $1921^{2}$. La maggior parte delle traduzioni del celebre umanista italiano le dobbiamo invece a Julia Dickstein-Wieleżyńska, un'importante divulgatrice della cultura italiana nel primo Novecento. Le sue versioni dei quattro componimenti delle Rime (V, XVI, CXXI, CXXIX) e di un componimento incluso nel primo libro degli Asolani (I 14) sono state pubblicate su Przeglad Humanistyczny nel 1931. Le traduzioni di Dickstein-Wieleżyńska sono un esempio di un crescente interesse per la poesia rinascimentale ${ }^{3}$. La loro seguente analisi semantico-lessicale sarà approfondita dalla comparazione con alcune traduzioni delle poesie di Petrarca realizzate da Faleński.

\section{JULIA DICKSTEIN-WIELEŻYŃSKA}

Julia Dickstein-Wieleżyńska ${ }^{4}$ (1881-1943) studiò a Berlino, a Roma e discusse la tesi di dottorato presso l'Università Giovanni Casimiro a Leopoli per poi insegnare all'Università libera di Polonia (Wolna Wszechnica Polska). Questa studiosa modernista spaziava tra vari interessi e argomenti di critica letteraria e filosofia. Era inoltre una poetessa talentuosa, autrice di tre raccolte liriche: Na duszy mej palecie (1919), Okiść (1928), Przed jego wielkim świattem (1935). Possedeva anche straordinarie capacità linguistiche. Conosceva dodici lingue tra cui il francese, lo spagnolo, l'italiano e il tedesco, che parlava correntemente (Płomieński, 1956, p. 273). Come osserva Mikołaj Sokołowski (2017, p. 499), "per lucidità e spessore intellettuale può essere considerata una delle più illustri menti dei primi decenni del Novecento in Polonia".

2 Nelle due traduzioni Grossekowa rinuncia alla partitura ritmica del testo originale. Nel sonetto LX utilizza l'endecasillabo, nel sonetto CLI invece lo sostituisce con il tredecasillabo.

3 Un'altra testimonianza dell'interesse per la letteratura rinascimentale negli anni Venti del XX secolo è il progetto Biblioteka włoska dra Edwarda Boyé. In questa serie Boyé ha pubblicato le proprie traduzioni di Boccaccio, Aretino e Machiavelli (Miszalska et al., 2007, p. 27).

4 Recentemente in Polonia si è aperta una fase di grande interesse per DicksteinWieleżyńska. Negli ultimi anni sono stati pubblicati almeno tre saggi su questa traduttrice (Sokołowski, 2017; Trzeciak-Cygan, 2017; Wilczak, 2014). 
Il talento per le lingue e la passione per la poesia di DicksteinWieleżyńska emergono dal suo lavoro traduttivo. Rendeva in polacco soprattutto la poesia italiana e divenne famosa grazie alle sue versioni dei componimenti di Giovanni Pascoli, Giosuè Carducci e Giacomo Leopardi. Traduceva anche i poeti italiani del Rinascimento 5 . Vanno menzionate pure le sue traduzioni di autori inglesi (Arthur Conan Doyle, Rabindranath Tagore) e francesi (Louise Ackermann). È importante sottolineare che gli interessi traduttivi di Dickstein-Wieleżyńska erano molto vari. Traduceva tra l'altro testi filosofici, come per esempio Einleitung in die Philosophie di Wilhelm Jerusalem. Tradusse inoltre dal polacco in italiano opere di Tadeusz Miciński (cf. Płaszczewska, 2010b, pp. 158-173) e di Henryk Sienkiewicz (Kleiner, 1946, p. 409).

In quanto critica letteraria Dickstein-Wieleżyńska si concentrava principalmente sulle "due letterature più vicine e più care", come definiva la letteratura italiana e quella polacca ${ }^{6}$. Il frutto di questi interessi è il ciclo di saggi Idee $i$ twórcy, che in gran parte presenta delle osservazioni riguardo a queste due letterature senza però prendere in considerazione l'epoca rinascimentale. Juliusz Kleiner descrive il lavoro critico letterario di Dickstein-Wieleżyńska come "oscillante fra la negazione ribelle e le fantasie mistiche" che e provare a sostenere le proprie tesi, propone libere riflessioni e le concentra in una cornice filologica (Kleiner, 1946, p. 409). Impiegando il metodo comparativo, cerca di interpretare la letteratura attraverso le idee filosofiche che essa contiene (Płaszczewska, 2010a, pp. 200, 592).

Dickstein-Wieleżyńska era attiva anche sul fronte sociale, essendo coinvolta soprattutto nel movimento femminista (cf. Walczak, 2014, pp. 121-132) e nell'aiuto ai prigionieri politici.

5 Per esempio Ludovico Ariosto, Giordano Bruno, Michelangelo Buonarroti, Vittoria Colonna, Tommaso Campanella.

6 "dwie najbliższe mi i najmilsze literatury: polska i włoska" (Dickstein, 1918, p. III).

7 "oscylujące między buntowniczą negacją a fantazjami mistycznymi" (Kleiner, 1946, p. 410). 


\section{TRADUZIONI BEMBIANE DI DICKSTEIN-WIELEŻYŃSKA}

\section{$\mathrm{V}$}

Czuprynka w lokach, niby jedwab miękki, Co się nad śniegiem wichrzy złoto-płowa, Oczy tak jasne, że aż noc się chowa

Przed ich rozblaskiem w różaność jutrzenki,

Śmiech, co łagodzi ostre bólu szczęki, Rubiny, perły - skąd spływają słowa, Słodkie, iż dusza świata zbyć gotowa; Biorąca serca w niewolę biel ręki,

Śpiew, w który boska harmonja się wciela, Rozum dojrzały już w dziecięce lata, Wdzięk nie widziany przez oczy niczyje,

Blask, w którym piękność i godność się brata, Ten to mój znęcił płomień. Czar w nich żyje, Przez niebo jeno dawan dla niewielu.

(Wieleżyńska, 1931, p. 116)
Crin d'oro crespo e d'ambra tersa e pura, ch'a l'aura su la neve ondeggi e vole, occhi soavi e più chiari che ' 1 sole, da far giorno seren la notte oscura,

riso, ch'acqueta ogni aspra pena e dura, rubini e perle, ond'escono parole sì dolci, ch'altro ben l'alma non vòle, man d'avorio, che i cor distringe e fura,

cantar, che sembra d'armonia divina, senno maturo a la più verde etade, leggiadria non veduta unqua fra noi, giunta a somma beltà somma onestade, fur l'esca del mio foco, e sono in voi grazie, ch'a poche il ciel largo destina. (Bembo, 1966, p. 510)

\section{XVI}

Moja urocza dzieweczka aniołek,

Z piosnką, co dawnych syren dźwięcznym śpiewem, Na miłej trawce pod cienistym drzewem

Śród godnych krasą siadła przyjaciółek.

$\mathrm{Na}$ ten widok strwożę. -

Sądziłem, żem jest w niebo uniesiony,

Tak słodyczy zasłony

Na moje oczy blask rzuciły błogi.

I już mówiłem: „O gwiazdy, o bogi,

O ty śpiewny przestworze..."

Gdym nagle ujrzał, że to są dziewice, Wesołe, cudnolice. -

Nie kajam się, Amorze,

Iże mię twoja strzała ugodziła, Jeśli maleńki twój dar ten raj zsyła.

(Wieleżyńska, 1931, p. 116)

La mia leggiadra e candida angioletta, cantando a par de le Sirene antiche, con altre d'onestade e pregio amiche sedersi a l'ombra in grembo de l'erbetta vid'io pien di spavento: perch'esser mi parea pur su nel cielo, tal di dolcezza velo avolto avea quel punto agli occhi miei. E già dicev'io meco: o stelle, o dei, o soave concento! Quand'i' m'accorsi ch'ell'eran donzelle, liete, secure e belle.

Amore, io non mi pento d'esser ferito de la tua saetta, s'un tuo sì picciol ben tanto diletta. (Bembo, 1966, pp. 518-519) 


\section{CXXI}

Ziemio, nieb ongi wypieszczona słowy,

Okuta w skały i wtulona w morza,

Ziemio nad wszystkie słoneczna i hoża,

Przez słup Apenin w dwie ścięta połowy,

Cóż, że cię niegdyś dzielny lud marsowy

Panią uczynił i lądów i morza,

Dawny się rab twój dziś bojem rozsroża,

Dłoń wsuwa w kosy stargane twej głowy.

O biada! Nawet rodzone twe dzieci, Ażeby nędzę twą wychylić do dna, Mieczem twe cudne przebijają ciało.

Boże! Gdzie niegdyś tyle cnów jaśniało, Taka ku Tobie dziś ohyda świeci, O wieku zbrodni, o raso wyrodna!

(Wieleżyńska, 1931, p. 117)

\section{CXXIX}

Gdy, Hieronimie, z cnoty Kwirynowi, Któremu Roma święte śćmiła życie,

Chcecie dorównać, wierząc, że w odkwicie,

Lecz mniej się srogo jego los ponowi,

Iż ten, co wokoło was i mnie się mrowi

Tłum się szlachecki nie kwapi w zachwycie Wieść was ku stolcom, o których marzycie, Lub drogę kwiecić waszemu losowi,

Nie dzerzcie ${ }^{*}$ żalu, Jest sławą jedyną, Być godnym cześci. - Tej nie zginąć chwale! Reszta jest dymem i nędzną pierzyną.

A nie najlepszym przypada w udziale. To kwiaty, co się na wietrze rozwiną, Albo śnieg biały w ciepłej słońca strzale. (Wieleżyńska, 1931, p. 117)
$\mathrm{O}$ pria sì cara al ciel del mondo parte, che l'acqua cigne e ' 1 sasso orrido serra, o lieta sovra ogni altra e dolce terra, che ' 1 superbo Appennin segna e diparte,

che giova omai, se '1 buon popol di Marte ti lasciò del mar donna e de la terra? Le genti a te già serve or ti fan guerra, e pongon man ne le tue treccie sparte.

Lasso, né manca de' tuoi figli ancora chi, le più strane a te chiamando, inseme la spada sua nel tuo bel corpo adopre.

Or son queste simili a l'antiche opre? $\mathrm{O}$ pur così pietate e Dio s'onora? Ahi secol duro, ahi tralignato seme! (Bembo, 1966, pp. 605-606)

Girolamo, se 'l vostro alto Quirino, cui Roma spense i chiari e santi giorni, cercate pareggiar, sì che ne torni men grave quel protervo aspro destino,

perché la nobil turba, onde vicino mi sete, a gradir voi lenta soggiorni, né v'apra a i desiati seggi adorni, a le civili palme anco il camino,

non sospirate: il meritar gli onori è vera gloria, che non pate oltraggio; gli altri son falsi e torbidi splendori

del men buon più sovente e del men saggio, che sembran quasi al vento aperti fiori, o fresca neve d'un bel sole al raggio. (Bembo, 1966, p. 612)

\section{* Ortografia originale}




\section{Gli Asolani I 14}

Gdy rozważam, Amorze,

Jako mię dręczysz i katujesz srodze,

Śmierci wprzeciw wychodzę,

Widząc, że krzywdom moim kres położę.

Lecz gdy stopa zabiega

Do mety wszelkich burzowych powodzi,

W duszy rozkosz się rodzi,

Krzepnie - więc niecham już drugiego brzega.

Życie w zgon mię przyzywa,

A śmierć na nowo do życia mię wpędza,

O nieskończona nędza!

Życie ją niesie, a śmierć jej nie zrywa.

(Wieleżyńska, 1931, p. 118)
Quand'io penso al martire,

Amor, che tu mi dai, gravoso e forte,

Corro per gir a morte,

Così sperando i miei danni finire.

Ma poi ch'i' giungo al passo,

Ch'è porto in questo mar d'ogni tormento,

Tanto piacer ne sento,

Che l'alma si rinforza, ond'io no 'l passo.

Così 'l viver m'ancide,

Così la morte mi ritorna in vita:

O miseria infinita,

Che l'uno apporta e l'altra non recide.

(Bembo, 1966, p. 341)

\section{ANALISI DELLE TRADUZIONI}

Per quanto riguarda l'aspetto metrico Dickstein-Wieleżyńska traduce tutti i sonetti usando l'endecasillabo e mantenendo lo schema rimico originale. Seguendo i testi di partenza, nelle quartine la traduttrice adotta le rime incrociate $\mathrm{ABBA}$ ABBA, mentre nelle terzine lascia, a seconda dei casi, le rime incatenate CDC DCD (sonetto CXXIX) oppure gli schemi spesso usati dai poeti italiani del Cinquecento, soprattutto da Vittoria Colonna, Giovanni della Casa e dallo stesso Pietro Bembo (Miszalska, 2010, p. 21; Miszalska, 2015, p. 361): CDE DEC (sonetto V) e CDE ECD (sonetto CXXI).

Il sonetto $\mathrm{V}$ è intriso di echi e riferimenti petrarcheschi. Il poeta rinascimentale presenta un catalogo di caratteri della donna perfetta, tutti presi da Petrarca. Descrive successivamente i capelli, gli occhi, il riso, le parole, la mano, il canto e l'intelletto dell'amante. Ad esempio, la descrizione degli occhi nella prima quartina contiene almeno quattro riferimenti alle composizioni del poeta laureatus. Gli occhi della donna descritta da Bembo sono soavi e più chiari che 'l sole. Anche gli occhi di Laura sono soavi (XXXVII 34, LXXIII 63, CCLIII 9) ${ }^{8}$. Questo ag-

8 Dionisotti confonde il sonetto CCLIII con il sonetto CCLII (Dionisotti, 1966, p. 510). 
gettivo Faleński di solito lo traduce come słodkie ${ }^{9}$, tranne che nel sonetto CCLIII dove occhi soavi vengono tradotti come czarowna twarz ${ }^{10}$. Dickstein-Wieleżyńska mantiene l'ordine in cui vengono descritti i caratteri femminili, rimanendo vicino al testo di partenza. La traduttrice omette la parola soavi, concentrandosi sul secondo epiteto, rinuncia al comparativo di maggioranza dell'aggettivo chiari e non rende la comparazione tra la chiarezza degli occhi e del sole. Dickstien-Wieleżyńska traduce occhi più chiari che 'l sole (espressione proveniente dal sonetto petrarchesco CCCLIII) come oczy tak jasne, że aż noc się chowa (occhi così chiari che la notte si nasconde) e continua questa iperbole usando l'enjambement. L'immagine degli occhi che possono far giorno seren la notte oscura del quarto sonetto di Bembo deriva dal verso petrarchesco (CCXV 13) dove il poeta descrive gli occhi di Laura che fanno chiara la notte, oscuro il giorno ${ }^{11}$. Nella scelta traduttiva di DicksteinWieleżyńska colpisce lo stile iperbolico. La traduttrice non trasmette la semplicità del linguaggio petrarchesco ${ }^{12}$, imitata da Bembo. Invece di mantenere la concisione della metafora del testo di partenza, sostituisce le semplici parole come sole o giorno con una metafora più elaborata, utilizzando dei termini sofisticati come rozblask (splendore) o różaność jutrzenki (il rosa dell'aurora) a differenza di Faleński che, traducendo il verso petrarchesco, rimane più vicino alla versione originale ( $z$ dnia noc, a z nocy jasne słońce wskrześnie - dal giorno la notte e dalla notte il chiaro sole risusciterà). In questo caso, dei cinque metodi di traduzione delle metafore correnti descritti da Newmark (1988, p. 96), DicksteinWieleżyńska applica quello che consiste nel "tradurre con un'immagine diversa che abbia lo stesso significato", Faleński invece quella che "consiste nel tradurre con una metafora che presenti la stessa immagine".

9 Tutte le traduzioni di Faleński citate in questo articolo, tranne il sonetto CCLII, provengono da Petrarca, 2005.

10 https://pl.wikisource.org/wiki/Pie\%C5\%9Bni_Petrarki/Sonet_215 Il sonetto CCLIII presente nella raccolta a cura di Salwa è stato tradotto da Agnieszka Kuciak.

11 L'origine di questo topos utilizzato spesso nella poesia romanza sono le Eroidi di Ovidio (XVI 320, XVIII 78) (Salwa, 2005, p. 715).

12 Miszalska (2000, p. 80) nota la stessa cosa riguardo alla traduzione di Faleński del sonetto I di Petrarca. 
Il verso successivo (riso, ch'acqueta ogni aspra pena e dura) contiene due reminiscenze petrarchesche (Dionisotti, 1966, p. 510). Nel sonetto XVII 5-6 il dolce riso di Laura calma i desideri del poeta, aspra pena e dura invece appare nella canzone LXXI 44. Faleński traduce il verbo acquetare come stać na straży (montare la guardia), spostando così l'attenzione sul complemento oggetto: gli ardenti miei desideri, tradotti come wrzace moje żadze (i miei bollenti desideri). Inoltre, il traduttore rende il verso più espressivo con la rima interna.

XVII, 5-6

Wprawdzie uśmiechy słodkie twe dziewicze

Stają u wrzących moich żądz na straży,
Vero è che 'l dolce mansüeto riso

Pur acqueta gli ardenti miei desiri,

(Petrarca, 1964, p. 17)

Dickstein-Wieleżyńska si allontana di meno dal testo di parten$\mathrm{za}$, traducendo acquetare come lagodzić. Sorprendente sembra invece la sua scelta di sostituire ogni aspra pena e dura con ostre bólu szczęki. La traduttrice ha introdotto il sostantivo szczęki (mascelle) assente nel testo fonte per conservare la rima, adoperando la tecnica della traduzione ritmica di André Lefevere (cf. Arduini \& Stecconi, 2007, p. 26). Dalla penna della traduttrice scaturisce l'immagine del dolore che affligge una persona sofferente. La traduzione di aspra pena e dura non si trova nella versione della canzone petrarchesca tradotta da Faleński che omette completamente questa parte. Faleński e Dickstein-Wieleżyńska raggiungono lo stesso obiettivo, usando tecniche diverse. Tutti e due rendono più espressiva l'immagine stereotipata e comune presente nel testo fonte: Faleński lo fa usando la rima interna, Dickstein-Wieleżyńska invece introducendo una metafora efficace.

Nell'ultimo verso della seconda quartina (man d'avorio, che $i$ cor distringe e fura) troviamo almeno quattro tracce dei componimenti di Petrarca (Dionisotti, 1966, p. 510). Anche la mano di Laura è del colore dell'avorio (CLXXXI 11) e distringe il cuore del poeta (CXCIX 1). Il verbo furare appare invece nelle canzoni XXIII 72 e CXXXV 25. Sia Petrarca che Bembo ricorrono al topos del cuore rubato che nella lirica romanza sottolineava la passività dell'amante e la dipendenza 
dell'amore da condizioni esterne ${ }^{13}$. Dickstein-Wieleżyńska sintetizza i due verbi distringere e furare nell'espressione brać w niewole (fare prigioniere). Modificando lievemente l'ordine grammaticale della frase, adopera la tecnica dello spostamento (Salmon, 2017, p. 219). Cambia anche il soggetto: nella sua versione è il colore dell'avorio della mano che opprime i cuori, non la mano del colore dell'avorio, come nel testo fonte.

Dickstein-Wieleżyńska non rende la ripetizione presente nel primo verso dell'ultima terzina (giunta a somma beltà somma onestade) e introduce la parola blask (splendore). Questo sostantivo e la parola rozblask dal quarto verso, simili di forma e significato, si ripetono spesso nelle versioni delle poesie bembiane della Dickstein-Wieleżyńska (il sonetto CXXI, la canzone XVI) e sono presenti, insieme ad altri termini dello stesso campo semantico, nei suoi propri componimenti poetici così frequentemente da poter definirli una delle dominanti della sua scrittura ${ }^{14}$. La traduttrice introduce talvolta il lessico collegato alla luminosità anche nelle traduzioni delle poesie di altri poeti italiani nonostante non sia presente nel testo fonte. Ci sono esempi di tale tendenza nelle sue versioni di Alla morte di Giuseppe Mazzini di Giosuè Carducci, dove la traduttrice inserisce il verbo lśnić (luccicare) senza che il suo corrispondente sia presente nel testo di partenza ${ }^{15}$, oppure della poesia All'Italia di Giacomo Leopardi dove l'idea espressa tra l'altro con l'aggettivo grande viene resa con il sostantivo blask ${ }^{16}$.

13 Anche in Dante, Voi che savete 2-4; A ciascun'alma presa 12-13 e nel sonetto CCLXXXVIII di Petrarca (Salwa, 2005, p. 502).

14 Il lessico collegato alla luce e allo splendore appare anche nella maggior parte delle poesie scritte da Dickstein-Wieleżyńska. Basta indicare un paio di esempi: oczy blaskiem zalane (Dickstein-Wieleżyńska, 1935, p. 8), oczy takie świetliste dziwy (Dickstein-Wieleżyńska, 1928, p. 25), przejasna chwila (Dickstein-Wieleżyńska, 1935, p. 10), jasność cudu (Dickstein-Wieleżyńska, 1935, p. 14).

15 Sí come d'un fatidico

Spirito tra il passato e l'avvenir, (Carducci, 1942, p. 70)

Iże duch jego wróży lśnit,

by dni minionych w przyszłe próg, (Dickstein-Wieleżyńska, 1935, p. 53).

16 Non dica, già fu grande, or non è quella? (Leopardi, 1974, p. 1).

Nie rzeknie: “z blasku jak spadta na nice!” (Leopardi, 1938, p. 4). 
Anche nella produzione di altri poeti polacchi del periodo interbellico si osserva una grande ricorrenza dei termini collegati all'idea dello splendore. Secondo Magdalena Amroziewicz (2019, p. 259) nella poesia di Czesław Miłosz nel periodo tra le due guerre l'elemento di fuoco (cf. Fiut 1981) e tutto quello che è associato con esso, cioè raggio, bagliore, splendore e luce, prevalevano in una maniera quasi ossessionante e insistente. Pure nella creazione poetica di Julian Tuwim la luminosità aveva un ruolo significativo (Smugała, 2003). Questo conduce a formulare l'ipotesi (da verificare in studi ulteriori) che la presenza del lessico che rimanda a un'idea di luminosità nella produzione di DicksteinWieleżyńska fosse una caratteristica dell'epoca piuttosto che un tratto individuale.

Nei primi versi del sonetto CXXI che ha per tema l'Italia divisa dalle guerre risulta amplificata la celebre definizione petrarchesca del Belpaese percorso dagli Appennini e circondato dalle Alpi (CXLVI 13-14). Seguono poi altre reminiscenze petrarchesche, fra cui il buon popol di Marte, definizione del popolo romano che Petrarca usò nei Trionfi (II 2). Dickstein-Wieleżyńska nella sua traduzione mantiene lo stile solenne del sonetto bembiano e la preoccupazione del poeta per la situazione politica del suo Paese. Il suo rimpianto risulta più forte nell'ultima terzina, dove pone domande retoriche esprimendo la nostalgia per gli anni di splendore della propria patria. Pur sostituendole con delle esclamazioni, la traduttrice è riuscita a rievocare il rimpianto commisto di rabbia e di rancore presente nel testo fonte. Quello che colpisce nell'ultima terzina sono i verbi jaśnieć (brillare) e świecić (splendere) non presenti nel testo di partenza. Essi rappresentano un altro esempio dell'uso di termini che rimandano a un'idea di luminosità.

Il sonetto CXXIX è dedicato a Girolamo Querini, patriarca veneziano negli anni $1524-1554^{17}$. Nella seconda strofa Bembo si riferisce alle difficoltà e ai fallimenti che il destinatario ha incontrato nella sua carriera politica. Il Non sospirate del verso nove è un riferimento al verso cinque del sonetto XXXVIII di Petrarca, a cui in gran parte è ispirata

17 Suo zio era Vincenzo Querini, un amico stretto di Bembo (Jedin, 1946, pp. 407424). 
la composizione di Bembo. La poesia di Petrarca è stata scritta come consolazione per Orso dell'Anguillara, che non poteva partecipare a un torneo, mentre Bembo nel suo sonetto consola Querini, turbato dai propri insuccessi politici. Il tema della vera gloria, svincolata dal giudizio degli altri, che troviamo nella seconda e nella terza strofa, è una reminiscenza dell'ode III di Orazio (Dionisotti, 1966, p. 613). Con l'ultima terzina Bembo mette le virtù antiche sullo stesso piano della bellezza della natura, ricorrendo alla filosofia umanistica.

Vale la pena mettere in evidenza alcune scelte operate dalla Dickstein-Wieleżyńska nella sua mirabile traduzione del sonetto bembiano. L'esclamazione petrarchesca non sospirate viene tradotta come nie dzierżcie żalu ${ }^{18}$ (non tenete rimpianto), così la traduttrice, introducendo il sostantivo żal (rimpianto), sottolinea ancora di più il pentimento del destinatario del sonetto. Gli altri son falsi e torbidi splendori Dickstein-Wieleżyńska lo rende con un più figurativo e concreto reszta jest dymem i nędzna pierzyna (il resto è un fumo e una misera coltre), allontanandosi dalla traduzione letterale che, come ha scritto Rosario Portale (2015, p. 310), "non è altro che una sterile fatica che snatura la bellezza e la profondità concettuale di un testo. [...] è quasi sempre arbitraria e non priva di rischi e forzature". Del men buon [...] e del men saggio dell'ultima terzina viene ridotto a nie najlepszym (non il migliore). La traduttrice attua in questo modo la tecnica della condensazione imposta dai vincoli metrici dell'endecasillabo (Salmon, 2017, p. 216).

Oltre ai tre sonetti Dickstein-Wieleżyńska traduce anche la canzone $^{19}$ XVI dalle Rime. La poesia è composta da una strofa di quindici versi, brevi e lunghi, dallo schema rimico ABBACDDEECFFCGG. Nei versi lunghi Bembo usa l'endecasillabo o il tredecasillabo, nei versi brevi il settenario. Dickstein-Wieleżyńska mantiene lo schema rimico originale ed è più accurata di Bembo nel considerare la lunghezza dei versi. Rispetta con precisione la loro lunghezza di sette o undici sillabe,

18 Felicjan Faleński ha sviluppato non sospirate di Petrarca traducendolo come przeciwnościom serce staw z opoki, il che rappresenta un esempio interessante di modulazione (Petrarca, 2005, p. 146).

19 Questo componimento si potrebbe classificare come barzelletta o frottola, una forma di canzone popolare nel Rinascimento (Kostkiewiczowa, 1998, p. 61). 
mentre l'autore dell'originale non sempre bada alla quantità delle stesse in ogni verso. La canzone, come tutto il secondo libro degli Asolani, fornisce un quadro degli amori allegri in uno scenario idilliaco. Angioletta che appare nel primo verso della canzone e che ritroviamo anche in due sonetti di Petrarca (CVI 1 e CCI 11), proviene dalla poetica del dolce stil novo ed è un termine caratteristico soprattutto per la poesia di Lapo Gianni $^{20}$. Bembo fa riferimento alla tradizione letteraria stilnovista, ma nello stesso tempo inserisce un elemento umanista: evoca il canto de le Sirene antiche. Dickstein-Wieleżyńska è riuscita perfettamente a trasmettere il significato delle parole chiave per il dolce stil novo. Il termine angioletta l'ha tradotto usando due parole: dzieweczka aniołek (fanciulla angioletta), sottolineando così l'innocenza e la frivolezza che caratterizzano la protagonista della canzone. Faleński, nella versione del sonetto CVI di Petrarca, ha tradotto l'espressione angioletta come dziewica rajska (vergine paradisiaca), attribuendole più serietà e dignità di Dickstein-Wieleżyńska, che sceglie di evidenziare la leggera e lieta atmosfera della canzone di Bembo. Tuttavia, nessuno dei due traduttori è riuscito a rendere il termine petrarchesco con una sola parola. Probabilmente, proprio per mantenere le undici sillabe nell'incipit, DicksteinWieleżyńska ha ridotto i due epiteti: leggiadra e candida a urocza (graziosa) usando di nuovo la condensazione. Questa operazione mostra che il principio base della traduzione non è stato l'accurata riproduzione della forma del testo fonte, imperfetta dal punto di vista metrico, ma il rispetto per la quantità di sillabe in ogni verso presupposta dalla traduttrice. Un altro termine caratteristico per il dolce stil novo è onestade che Dickstein-Wieleżyńska adeguatamente traduce come godność (cf. Miszalska, 2001/2002, pp. 30-43).

Dickstein-Wieleżyńska è l'unica a tradurre la poesia di Bembo non compresa nella raccolta Rime: produce la versione di una canzone di dodici versi inclusa nel primo libro degli Asolani. Anche in questa traduzione la traduttrice è fedele allo schema delle rime incrociate ABBACDDCEFFE e coerente nell'uso del settenario per quanto riguar-

20 Angioletta in sembianza 1-2, Dolc'è 'l pensiero 5-7 (Salwa, 2005, p. 592). 
da i versi originali di sette sillabe e all'uso dell'endecasillabo per i versi in dodici sillabe.

La canzone riguarda le riflessioni di Perottino sulla gioia che potrebbe portare la morte in quanto liberazione per l'amante sofferente. Wieleżyńska è riuscita a ottenere l'equifunzionalità semantica grazie alla precisione linguistica e alla capacità di trovare i traducenti accurati delle parole chiave che rendono l'atmosfera del componimento disperata e amplificano il dolore del protagonista (per esempio: corro per gir a morte - śmierci wprzeciw wychodzę, così 'l viver m'ancide - zycie $w$ zgon mię przyzywa, o miseria infinita - o nieskończona nędza!).

\section{CONCLUSIONI}

Mantenere tutti i rigori formali del genere letterario senza allontanarsi troppo dallo strato semantico e stilistico è una vera sfida per i traduttori dei sonetti (Woźniak, 2001-2002, p. 14). Da questa analisi risulta che Dickstein-Wieleżyńska è riuscita a realizzare questo compito. Nelle sue versioni dei sonetti bembiani la traduttrice usa sempre l'endecasillabo e mantiene lo schema rimico originale ${ }^{21}$. Dalle sette strategie di traduzione poetica rilevate da Lefevere $(1975$, p. 9) Dickstein-Wieleżyńska adopera quella della traduzione ritmica che conserva lo schema rimico e il metro del testo fonte. Adottando le questioni metriche e rimiche come l'idea di fondo, Dickstein-Wieleżyńska attua perlopiù la tecnica della condensazione, indispensabile dove è vincolante il numero delle parole o sillabe (Salmon, 2007, p. 216).

Le sue traduzioni sono in gran parte equivalenti dal punto di vista semantico. Sebbene la traduttrice introduca qualche riduzione o amplificazione, lo fa senza sconvolgere la dominante. Colpisce l'introduzione nelle traduzioni delle poesie bembiane dei termini che rimandano a un'idea della luminosità che sono una delle caratteristiche della produzione poetica di Dickstein-Wieleżyńska. Si nota tra l'altro una certa tendenza

${ }^{21} \mathrm{Al}$ contrario di Grossekowa che utilizza sia l'endecasillabo che il tredecasillabo e modifica le rime a seconda della pratica traduttiva di sonetti in polacco che permette l'uso di tutte e due le metriche (cf. Miszalska, 2000, pp. 80-81). 
di creare una traduzione poetica attraverso metafore piuttosto espressive che a tratti fanno perdere la semplicità che caratterizza lo stile origina$1 \mathrm{e}^{22}$. D'altra parte Dickstein-Wieleżyńska traducendo la canzone XVI trova plausibili equivalenti delle parole chiave riconducibili al dolce stil novo. La traduttrice non è però ossessivamente attaccata all'idea di tradurre letteralmente tutti i termini e non esita a proporre le proprie versioni dei versi bembiani, mostrando così che "traduzione è interpretazione e interpretazione è creazione o ri-creazione, di uno stile che [...], deve cercare di restituire il senso e il messaggio autentico del testo stesso" (Portale, 2015, p. 310).

\section{BIBLIOGRAFIA}

Amroziewicz, M. (2019). „Promień ostry, który nas otwiera”. Światło w międzywojennej poezji Czesława Miłosza. Poznańskie Studia Polonistyczne. Seria Literacka, 36 (56), 259-288.

Arduini, S., \& Stecchoni, U. (2007). Manuale di traduzione. Teorie e figure professionali. Roma: Carocci.

Bembo, P. (1966). Prose della volgar lingua, Gli Asolani, Rime (edited by C. Dionisotti). Torino: Editori Associati S.p.A.

Bembo, P. (2019). Stance (B. Sosnowska, Trans.). Warszawa: Wydawnictwo Naukowe Sub Lupa.

Carducci, G. (1942). Giambi ed epodi. Bologna: Zanichelli.

Dickstein, J. (1918). Idee i twórcy. Warszawa: Tow. Akc. S. Orgelbranda Synów. Dickstein-Wieleżyńska, J. (1928). Okiść. Warszawa: Księgarnia F. Hoesicka.

Dickstein-Wieleżyńska, J. (1931). Czupryna w lokach niby jedwab miękki; Hieronimi, z cnoty Kwirynowi; Ziemio, niech ongi wypieszczona słowy; Moja urocza dzieweczka aniołek. Przeglad Humanistyczny, 6(1), $116-118$.

Dickstein-Wieleżyńska, J. (1935). Przed jego wielkim świattem. Warszawa: Dom Książki Polskiej.

Dionisotti, C. (1966). Note. In P. Bembo, Prose della volgar lingua, Gli Asolani, Rime (edited by C. Dionisotti). Torino: Editori Associati S.p.A.

Fiut, A. (1981). „W mowie ognia...” (Wokół poezji Czesława Miłosza). Ruch Literacki, 1 (124), 1-14.

${ }^{22}$ Questa attitudine è presente anche nelle versioni di Grossekowa. 
Grossek-Korycka, M. (1921). Gdy ustępując miejsca, z nieba schodzi słońce; Rymy szczęśliwe, lekkostropne zwrotki. In A. Lange, \& A. Tom (Eds.) Panteon literatury wszechświatowej. Italja (p. 87). Warszawa: Polska Składnica Pomocy Szkolnych.

Jedin, H. (1946). Vincenzo Quirini und P. Bembo. Miscellanea Giovanni Mercati, VI, 407-424.

Kleiner, J. (1946). Julia Wieleżyńska. Pamiętnik literacki, 36(3-4), 408-411. Kostkiewiczowa, T. (1998). Barzelletta. In J. Sławiński (Ed.), Słownik terminów literackich (p. 61). Wrocław-Warszawa-Kraków: Wydawnictwo Ossolineum.

Kostkiewiczowa, T. (1998). Frottola. In J. Sławiński (Ed.), Słownik terminów literackich (pp. 167-168). Wrocław-Warszawa-Kraków: Wydawnictwo Ossolineum.

Lefevere, A. (1975). Translating Poetry, Seven Strategies and a Blueprint. Amsterdam: Van Gorcum.

Leopardi, G. (1938). Poezje (J. Dickstein-Wieleżyńska, Trans.). Warszawa: Instytut Wydawniczy "Bibljoteka Polska".

Leopardi, G. (1974). Canti. Milano: Rizzoli.

Miszalska, J. (2000). Jeszcze o przekładzie „Sonetu I” Petrarki. Przekładaniec, $7,80-88$.

Miszalska, J. (2001/2002). Jak thumaczyć dantejski sonet XV [Tanto gentile e tanto onesta pare]?. Przekładaniec, 9, 30-43.

Miszalska, J., Gurgul, M., Surma-Gawłowska, M., \& Woźniak, M. (2007). Od Dantego do Fo. Włoska poezja i dramat w Polsce (od XVI do XXI wieku). Kraków: Collegium Columbinum.

Miszalska, J. (2010). Sonet w Polsce od XVI do początków XIX wieku a przekłady z języka włoskiego. Italica Wratislaviensia, 1, 2010, 16-33.

Miszalska, J. (2015). Z ziemi włoskiej do Polski... Przekłady z literatury włoskiej w Polsce do końca XVIII wieku. Kraków: Collegium Columbinum. Miszalska, J. (2020). Działalność tłumaczek a polski kanon literatury włoskiej: Waleria Marrené-Morzkowska i Zofia Ernstowa. Porównania, 1 (26), 139-156.

Newmark, P. (1988). La traduzione: problemi e metodi. Milano: Garzanti.

Olech, B. (2005). Wieszczka wydm niebieskich... In M. Grossek-Korycka, Utwory wybrane (pp. 5-62). Kraków: Wydawnictwo Literackie.

Petrarca, F. (1964). Canzoniere (edited by G. Contini). Torino: Einaudi.

Petrarca, F. (2005). Drobne wiersze wtoskie. Rerum vulgarium fragmenta (edited by P. Salwa). Gdańsk: słowo/ obraz terytoria. 
Płaszczewska, O. (2010a). Przestrzenie komparatystyki - italianizm. Kraków: Wydawnictwo Uniwersytetu Jagiellońskiego.

Płaszczewska, O. (2010b). O włoskich przekładach poezji Tadeusza Micińskiego. Italica Wratislaviensia, 1, 158-173.

Płomieński, J. E. (1956). Twórcy bez masek. Warszawa: Pax.

Portale, R. (2015). Translation for Translation's Sake: Problemi, considerazioni ed esperienze di un "traduttore-artigiano". Kwartalnik Neofilologiczny, LXII, 309-319.

Porębowicz, E. (1890). Tutaj spojrzała; Sąż to te piękne oczy. In E. Porębowicz, \& J.A. Święcicki (Ed.), Dzieje literatury powszechnej, vol. 3 (p. 91). Warszawa: S. Lewentel.

Salmon, L. (2017). Teoria della traduzione. Milano: Franco Angeli.

Salwa, P. (2005). Noty i komentarze. In F. Petrarca, Drobne wiersze włoskie. Rerum vulgarium fragmenta (pp. 477-867). Gdańsk: słowo/obraz terytoria.

Smugała, K. (2003). Światło jako znak genesis w liryce Juliana Tuwima. Rocznik Towarzystwa Literackiego imienia Adama Mickiewicza, 38, $39-52$.

Sokołowski, M. (2017). Attraverso Słowacki. Sulle traduzioni carducciane di Julia Dickstein-Wieleżyńska. Kwartalnik Neofiloloficzny, LXIV, 497-505.

Trzeciak-Cygan, M. (2017). Una femminista traduce Leopardi: il caso di Julia Dickstein-Wieleżyńska. Rivista di letteratura italiana, 35 (2), 83-91.

Woźniak, M. (2000). „Voi ch'ascoltate in rime sparse il suono” Francesca Petrarki: jak przełożyć sonet-słowo wstępne do „Canzoniere”? Przekładaniec, 7, 69-79.

Woźniak, M. (2001-2002). Przyjemności i pożytki tłumaczenia dawnej poezji włoskiej. Przekładaniec, 9, 9-29.

Wilczak, M. (2014). „Feministka starego kroju” - Julia Dickstein-Wieleżyńska wobec kwestii kobiecej. Bibliotekarz Podlaski, 15(1), 121-132.

Riassunto: Il presente articolo si pone l'obiettivo di analizzare le traduzioni polacche novecentesche di Pietro Bembo. Anche se Bembo è uno dei principali rappresentanti del Rinascimento italiano e uno scrittore prolifico, di tutte le sue liriche sono state tradotte integralmente soltanto sei canzoni dalla raccolta Rime, una breve poesia dal dialogo Gli Asolani e recentemente le Stanze. La maggior parte delle traduzioni le dobbiamo a Julia Dickstein-Wieleżyńska, importante divulgatrice della cultura italiana nel periodo interbellico. Due poesie sono state tradotte da Maria Grossekowa, una poetessa, pubblicista e femminista dei primi del Novecento. Bisogna menzionare anche i piccoli frammenti dei sonetti di Bembo tradotti da Edward Porębowicz. L'articolo si concentra sull'analisi metricoformale e semantico-lessicale delle versioni di Dickstein-Wieleżyńska ed esamina le tecniche usate 
dalla traduttrice. Le traduzioni sono piuttosto equivalenti dal punto di vista semantico. Sebbene la traduttrice introduca qualche riduzione o amplificazione, lo fa senza sconvolgere la dominante. Inoltre, è stato notato che Dickstein-Wieleżyńska nelle sue traduzioni spesso utilizza i termini che rimandano a un'idea di luminosità e che rappresentano anche una delle dominanti della sua produzione poetica. Poiché Bembo è considerato il pioniere del petrarchismo, l'analisi delle traduzioni polacche dei componimenti bembiani viene approfondita dalla comparazione con alcune versioni delle poesie di Petrarca realizzate da Felicjan Faleński e pubblicate nel 1881.

Parole chiave: Bembo, Rinascimento, traduzione, Dickstein-Wieleżyńska, Grossekowa 